\title{
Evaluation of the impact of limited-sequence MRI brain protocol (fast brain MRI) on diagnostic accuracy and length of hospital stay for patients with stroke-like symptoms
}

\author{
Authors: Gauravsingh Dhunnoo ${ }^{A}$ and Diptarup Mukhopadhyay ${ }^{A}$
}

\section{Introduction}

A significant number of patients are admitted under the stroke team with symptoms mimicking cerebrovascular events. Their diagnosis can be challenging, delaying discharges and impacting management. This imposes a significant burden on bed spaces and incurs significant costs to the NHS.

We aim to review the use of 'fast brain' magnetic resonance imaging (MRI) in patients admitted with stroke-like symptoms and to assess their impact as a diagnostic aid in the management of the patients, including initiation of new medications and facilitating discharge.

\section{Method}

We collected data on the stroke unit at Princess of Wales Hospital, Bridgend. The data were extracted retrospectively from the admission registry for the stroke ward as well as from the discharge summaries on the health board's clinical portal. The Synapse system was used to get results of CT of the head and MRI of the brain during the admission. The length of stay between 2016 and 2017 was compared (2017 being the year when MRI of the brain were used to help in the diagnosis of stroke in patients admitted to the stroke unit).

\section{Results/analysis}

During 2016, 627 patients were admitted to the stroke unit at Princess of Wales hospital. The average length of stay (LOS) was 9.4 days. The number of admissions to the stroke unit in 2017 was 665 . The average LOS was 8 days. This was a $15 \%$ reduction in LOS in 2017 compared to 2016 (Fig 1).

Of the 665 admissions in 2017, 159 patients had fast brain MRI to help confirm their diagnosis. The average waiting time for the scan was 2.7 days with $36.5 \%$ having their scan on day one after admission. The average LOS was 6.5 days, with $72 \%$ being discharged within 5 days of admission (Fig 1).

All patients except one had a head scan on admission $(\mathrm{n}=158) .13$ patients had CT reported as a new or recent infarct. Of those seven (54\%) had a confirmed infarct on MRI and

Author: APrincess of Wales Hospital, Bridgend, UK (a)

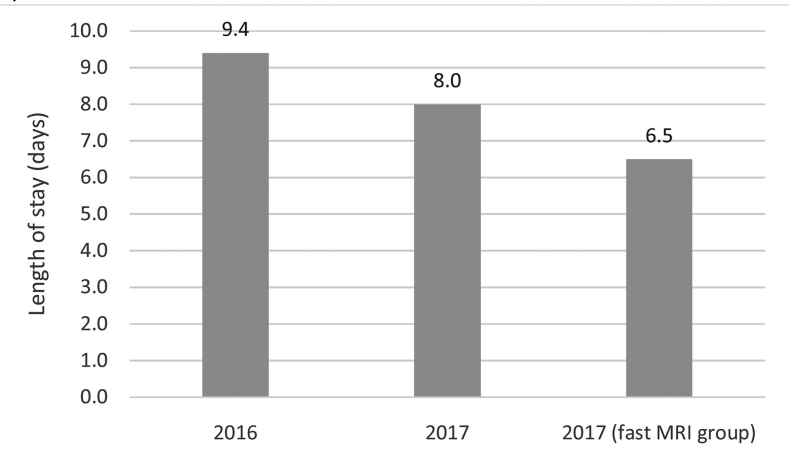

(b)

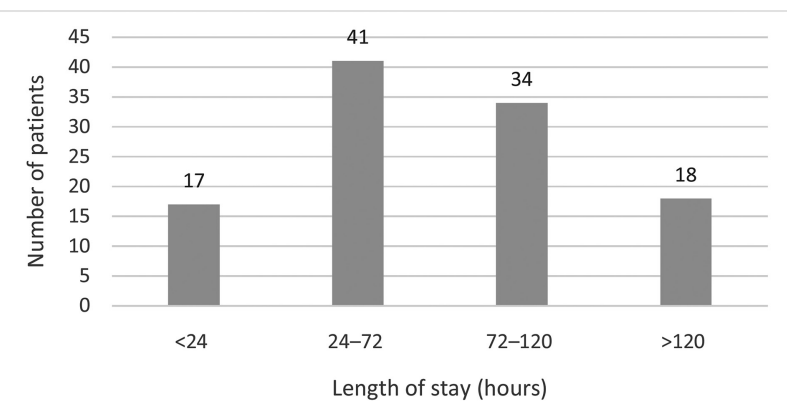

Fig 1. Length of hospital stay. a) Change in length of hospital stay. b) Length of stay in magnetic resonance imaging group.

six $(46 \%)$ had a recent infarct excluded. Of the patients with negative CT of the head for new/recent infarct, 56 (38.6\%) had a confirmed new event on fast brain MRI.

$75 \%$ of patients with positive MRI were started on clopidogrel, $11 \%$ had a new diagnosis of atrial fibrillation, and $10 \%$ were anticoagulated on discharge.

\section{Conclusions}

There was a $31 \%$ reduction in LOS in the MRI group compared to the average LOS in 2016 on the stroke ward with 461 bed-days saved. This amounted to $£ 207,450$ saved in 2017 . The 
Anticoagulated following discharge

Newly started on clopidogrel

Previously on clopidogrel

Previously on aspirin

Anticoagulated

Newly diagnosed AF

Previously known to have AF

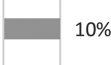

\begin{tabular}{|l|}
\hline \\
\hline
\end{tabular}

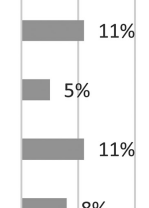

$8 \%$

$\begin{array}{lllllllll}0 \% & 10 \% & 20 \% & 30 \% & 40 \% & 50 \% & 60 \% & 70 \% & 80 \%\end{array}$

Fig 2. Group with positive magnetic resonance imaging, giving details on change of management strategy.
MRI was superior to CT of the head in the diagnosis of new infarcts. Early specialist stroke input and early confirmation of diagnosis reduced the length of stay, saving on the cost of beds and staffing on the stroke ward, and allowed risk stratification for future stroke events. This impacted the medical management of stroke-positive patients, including the introduction of anticoagulation in patients with atrial fibrillation and initiation of clopidogrel when appropriate (Fig 2).

\section{Conflicts of interest}

None declared. 